\title{
EDITORIAL
}

\section{Promoting effective self-management programmes to improve COPD}

\author{
J. Bourbeau* and J. van der Palen\#
}

$\mathbf{T}$ he recent priority focus on integrated chronic care is not surprising given the burden of chronic disease. This focus has led to the development of integrated care programmes for chronically ill patients. Despite important heterogeneity in these programmes, there is a commonality of objectives i.e. to reduce fragmentation of care, and to increase continuity and coordination of care [1]. Self-management interventions are an important component of integrated chronic care and the most commonly mentioned [2].

An increasing number of healthcare professionals agree that patients with chronic disease should receive support to help them self-manage their disease as effectively as possible. Current evidence in chronic diseases suggests that patients with effective self-management skills make better use of healthcare professionals' time and have enhanced self-care [3, 4]. Through their daily decisions about medication, selfmeasurements and exercise, people with chronic diseases play a central role in determining the course of their disease. Unfortunately, what is already common practice in other chronic diseases is not yet applied in chronic obstructive pulmonary disease (COPD). We now urgently need to tackle COPD and ensure that effective self-management interventions, as an important part of integrated chronic care, are implemented when present.

\section{COPD SELF-MANAGEMENT: THE EVIDENCE}

We now have sufficient scientific evidence to recommend selfmanagement interventions for patients with COPD. Two recent systematic reviews have shown positive outcomes for patients with COPD $[5,6]$. In the most recent update of the Cochrane Database of Systematic Reviews, it was demonstrated that self-management programmes reduce the probability of COPD-related hospital admissions [6]. Another systematic review demonstrated a significant reduction in healthcare utilisation (unscheduled/emergency centre visits, number of hospitalisations and length of hospital stay) in trials that implemented self-management with other components of the

*Respiratory Epidemiology and Clinical Research Unit, Montreal Chest Institute of the Royal Victoria

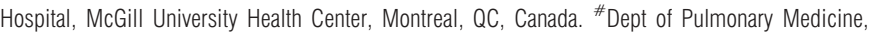
Medisch Spectrum Twente, Enschede, The Netherlands.

STATEMENT OF INTEREST: A statement of interest for $\mathrm{J}$. Bourbeau can be found at www.erj.ersjournals.com/misc/statements.shtml

CORRESPONDENCE: J. Bourbeau, Respiratory Epidemiology and Clinical Research Unit, Montreal Chest Institute, 3650 St Urbain, Montreal, QC, Canada H2X 2P4. Fax: 1 5148432083. E-mail: jean.bourbeau@mcgill.ca chronic care model compared to trials with self-management alone [5]. Self-management also holds promise of positive economic benefits in light of increased patient case loads and rising costs of hospitalisations [7], but more evidence is urgently needed.

We may be enthusiastic about recent results but there remains uncertainty about what aspects of self-management programmes are most effective, mainly due to the wide variety in the way self-management programmes are designed, delivered and evaluated. In the past, failure to recognise this wide variety has led not only to inappropriate conclusions about the effectiveness of these programmes but also to a high likelihood of inappropriate application of research results in clinical practice.

\section{SELF-MANAGEMENT PROGRAMMES ARE MORE THAN PATIENT EDUCATION}

Self-management interventions involve collaboratively helping patients acquire and practice the skills needed to carry out disease-specific medical regimens, change their health behaviour to adjust their roles for optimal function, improve day-today control of their disease, and improve their well-being. Self-management also includes assessment of progress and problems, goal setting, and problem-solving. While patient education is necessary, as it is essential that patients develop knowledge, alone it is insufficient. Patient education means giving patients information (materials and instruction) regarding their condition and possible management. In itself, education is not a self-management intervention, as it does not achieve patient behavioural change and does not let them acquire and practice skills. Therefore, the term "education" should be used with caution.

\section{ASSURING THE SUCCESS OF SELF-MANAGEMENT INTERVENTIONS}

Why have we had limited success with self-management studies? We have neglected to consider the complex sequence of effects resulting from self-management programmes. Selfmanagement programmes have many objectives and endpoints. Measuring only patients' visits to the emergency room or hospital admissions is overly narrow, and may not be realistic in the short term. The pivotal objective of selfmanagement programmes is to change patients' behaviour. Therefore, evaluations should start with measures of patients' behaviour and subsequently assess the change in behaviour. It is only after achieving behaviour change that we can expect proper self-management of the disease resulting in better 
patient outcomes and reduction in utilisation of healthcare services. Evaluating the benefits of a programme based solely on changes in patients' health status or the number of hospital admissions before evaluating changes in patients' behaviour, and determinants of this behaviour, such as self-efficacy, may result in false-negative results. In many studies, there may have been a failure to intervene, which resulted in a failure to yield structural behaviour change.

The success of a self-management programme should correspond to the goals of self-management i.e. acquiring key self-management skills and self-health behaviours [8]. Key selfmanagement skills include: resource utilisation; patientprovider relationships; problem solving; decision making; early symptom recognition; and taking action, based on a predefined action plan. As self-health behaviours are performed with success in various situations of everyday life, patients develop an improved sense of self-efficacy. Appropriate time and follow-up are essential if we want patients to increase their self-efficacy and change their health behaviour, which in turn leads to better disease control and improves patient outcomes. In addition, features of the selfmanagement programme will have to be tailored to patients' needs and concerns, disease severity and associated comorbidities that are common in COPD patients. Regular progress and problem assessments, goal setting, motivation and confidence building, and problem-solving support must be provided by an appointed health professional "case manager". The fact that aspects of a self-management programme have to be tailored to the individual does not mean self-management programmes need to be individual programmes. Small-group programmes are an attractive alternative, as patients can also learn from each other.

Self-management also refers to patients as having achieved the skills and the confidence to manage their health and engage in particular behaviours. For COPD patients, these self-health behaviours are pharmacological (adherence to medication, early recognition of symptoms and prompt access to early treatment in the event of exacerbations) and non-pharmacological (breathing techniques, exercising, nutritional programmes and stress management). Self-management is not intended to replace components of patient healthcare such as medication and pulmonary rehabilitation. For example, pulmonary rehabilitation is increasingly becoming a realistic component of COPD patient management but it should not stand as an isolated, albeit multidimensional intervention. It should be part of an integrated care process and include selfmanagement support i.e. aiming to achieve a shift from management by the healthcare provider, to management by the patients themselves, which implies structural behaviour change.

\section{CULTURAL SHIFT IN WORK PRACTICES AND SERVICE DELIVERY}

A major cultural shift in work practices and service delivery is required for self-management principles to be embedded throughout the continuum of chronic disease care. Engagement of health professionals and physicians is critical to the successful application of self-management programmes. Primary care should actively support patient self-care strategies but additional resources are often required. Secondary and tertiary care have to collaborate as programme content may need to be adapted to reflect the growing medical scientific knowledge on a given disease i.e. COPD, congestive heart failure $[9,10]$. Patients should be able to move from primary through secondary to tertiary care and back as part of a continuity of care model for patients. Self-management support programmes should have a flexible approach to both delivery and programme content. Support may be provided via a range of formats i.e. internet, telephone, mentoring or coaching, face-to-face individual or small-group-based activities. Self-management needs to be within and outside the primary care setting; it needs to take place as part of an integrated chronic care system to ensure continuity and coordination of care.

Training and protocols for case managers are often missing. Training is especially important since many appointed health professional case managers may not have the psychosocial skills to facilitate patients' behavioural change. Differentiating self-management support tasks and teaching skills should be considered. Some of the teaching skills may be disease-specific. Protocols ensure some degree of standardisation and consistency in the way the programme is delivered and in programme content. They also help enhance the case manager's training.

\section{LESSONS FOR CLINICAL PRACTICE}

Although self-management in COPD has been demonstrated to be successful in the reduction of health service utilisation (such as visits to the emergency room and hospital admissions), concrete strategies are needed as is the organisational capacity to implement them.

Lesson 1: Change your way of thinking. Healthcare providers have to reshape their thinking. Engagement and endorsement of healthcare professionals is critical to success.

Lesson 2: Do it for the right reason. Self-management-support programmes should be imbedded in the healthcare system (collaboration of the primary, secondary and tertiary care) but not only to relieve pressure on the healthcare system. The pivotal objective is to change patients' behaviour (medically and non-medically related) for better disease control and patient outcomes.

Lesson 3: Self-management is not only education. Programmes have to be patient-centred and tailored to the needs of patients i.e. patients with various comorbidities, disease severity and complexity, and different psychosocial situations. The best programmes are those with a flexible approach to both delivery and programme content. It also requires reinforcement and follow-up.

Lesson 4: Training is essential. Healthcare professionals must be trained to ensure proper skills i.e. psychosocial skills to facilitate patient behavioural change as well as specific evidence-based content skills relevant to COPD. An appointed health professional case manager responsible for guiding and coaching patients is the most effective and accepted way of achieving this.

Lesson 5: Be aware of pitfalls. There are many pitfalls: underestimating the difficulty of managing change, misjudging the skills of healthcare professionals and whether they are able to 
integrate this with their other roles, and being overly optimistic regarding timescales and potential results (under-estimating the complex sequence of effects of self-management support).

\section{FUTURE RESEARCH}

Better research is needed to increase our understanding of the relative effectiveness of specific components and how best to support chronic obstructive pulmonary disease patients in selfmanagement. The patient population has to be well described with respect to chronic obstructive pulmonary disease severity and comorbidities. Furthermore, people who enquire, enrol, attend and complete self-management programmes need to be characterised. Trials have to be properly powered and the measurement timeframe needs to match the timeframe in which the self-management support objectives (e.g. selfefficacy) and behaviour change can be attained. Self-management interventions have to be properly defined (case manager training, support to patients, programme content, protocols regarding programme delivery, communication with patients and with physicians). Selection of sensitive and appropriate outcomes is also essential; assessing process and behaviour change should be required as outcome measures. Patient selfefficacy and specific behaviours need to change before a reduction in the number of hospitalisations and costs can be achieved. Finally, very little is known about factors that both help and limit dissemination of these programmes in realworld settings.

\section{REFERENCES}

1 Ouwens M, Wollersheim $H$, Hermens R, Hulscher M, Grol R. Integrated care programmes for chronically ill patients: a review of systematic reviews. Int J Qual Health Care 2005; 17: 141-146.

2 Wagner EH. Chronic disease management: what will it take to improve care for chronic illness? Eff Clin Pract 1998; 1: $2-4$.

3 Lorig K, Sobel D, Stewart A, et al. Evidence suggesting that a chronic disease self-management program can improve health status while reducing hospitalization: a randomized trial. Med Care 1999; 37: 5-14.

4 Barlow JH, Turner AP, Wright CC. A randomized controlled study of the Arthritis Self-Management Programme in the UK. Health Educ Res 2000; 15: 665-680.

5 Adams SG, Smith PK, Allan PF, Anzueto A, Pugh JA, Cornell JE. Systematic review of the chronic care model in chronic obstructive pulmonary disease prevention and management. Arch Intern Med 2007; 167: 551-561.

6 Effing T, Monninkhof E, van dV, et al, Self-management education for patients with chronic obstructive pulmonary disease. Cochrane Database Syst Rev 2007; 1: CD002990.

7 Bourbeau J, Collet JP, Schwartzman K, Ducruet T, Nault D, Bradley C. Economic benefits of self-management education in COPD. Chest 2006; 130: 1704-1711.

8 Bourbeau J, Nault D, Dang-Tan T. Self-management and behaviour modification in COPD. Patient Educ Couns 2004; 52: 271-277.

9 Glouberman S, Mintzberg H. Managing the care of health and the cure of disease-Part II: Integration. Health Care Manage Rev 2001; 26: 70-84.

10 Glouberman S, Mintzberg H. Managing the care of health and the cure of disease-Part I: Differentiation. Health Care Manage Rev 2001; 26: 56-69. 